\title{
Promoter DNA methylation analysis reveals a combined diagnosis of CpG-based biomarker for prostate cancer
}

\author{
Yuanyuan Tang ${ }^{1, *}$, Shusuan Jiang ${ }^{2, *}$, Yinmin $\mathbf{G u}^{3}$, Weidong $\mathbf{L i}^{3}$, Zengnan $\mathbf{M o}^{2,4}$, \\ Yuanjie Huang ${ }^{3}$, Tianyu $\mathbf{L i}^{2,4}$ and Yanling $\mathrm{Hu}^{3,4,5}$ \\ ${ }^{1}$ Guangxi Reproductive Medical Research Center, First Affiliated Hospital of Guangxi Medical University, Nanning, Guangxi \\ 530021, China \\ ${ }^{2}$ Department of Urology, First Affiliated Hospital of Guangxi Medical University, Nanning, Guangxi 530021, China \\ ${ }^{3}$ Life Sciences Institute, Guangxi Medical University, Nanning, Guangxi 530021, China \\ ${ }^{4}$ Center for Genomic and Personalized Medicine, Guangxi Medical University, Nanning, Guangxi 530021, China \\ ${ }^{5}$ Guangxi Colleges and Universities Key Laboratory of Biological Molecular Medicine Research, Guangxi Medical University, \\ Nanning, Guangxi 530021, China \\ *These authors have contributed equally to this work \\ Correspondence to: Yanling Hu, email: ylhuteam@163.com \\ Tianyu Li, email: litianyu@gxmu.edu.cn
}

Keywords: prostate cancer, DNA methylation, promoter, diagnostic biomarker, gene

Received: October 20, $2016 \quad$ Accepted: February 28, $2017 \quad$ Published: March 22, 2017

Copyright: Tang et al. This is an open-access article distributed under the terms of the Creative Commons Attribution License 3.0 (CC BY 3.0), which permits unrestricted use, distribution, and reproduction in any medium, provided the original author and source are credited.

\section{ABSTRACT}

Background: Prostate cancer (PCa) is the most common tumor in elderly men. However, the specificity and sensitivity of serum prostate-specific antigen levels in PCa diagnosis are controversial. This study aims to reveal a novel diagnosis biomarker in PCa.

Materials and Methods: The differential methylated CpG sites between 423 primary PCa and 39 adjacent samples from The Cancer Genome Atlas (TCGA) on Illumina HumanMethylation 450 platform were analyzed. The diagnostic methylation markers were mined using the Prediction Analysis of Microarrays package in Bioconductor. Then, the Gene Expression Omnibus data was used for verification. Pyrosequencing was applied to improve methylation levels of five CpGs (cg06363129, cg08843517, cg05385513, cg07220448 and cg11417025).

Results: The area under curve of receiver operating characteristic of eight diagnostic methylation CpGs (cg06363129, cg08843517, cg03576469, cg05385513, cg07220448, cg11417025, cg20883831, and cg23824801) in TCGA data ranged from 0.910 to 0.939 . Except for cg20883831 and $\operatorname{cg} 23824801$, the correlations between methylation levels of six other sites and their expressions in patients were significant $(r>0.5$ and $P<0.001)$. The methylation level of cg06363129 was significantly different between the groups of Gleason Score (GS) $=7$ and GS $\geq 8$ ( $P<0.05)$. Pyrosequencing in our samples confirmed that four diagnostic methylation sites (cg06363129, cg08843517, cg05385513, and cg11417025) had high diagnostic efficacy.

Conclusions: The combined diagnosis of four methylation CpGs sites (cg06363129, cg08843517, cg05385513, and cg11417025) in the gene promoter has high tissue specificity and diagnostic efficacy for $\mathrm{PCa}$. Results revealed a novel potential biomarker for prostate cancer diagnosis. 


\section{INTRODUCTION}

Prostate cancer $(\mathrm{PCa})$ is the most common cancer in the male reproductive system and is the leading cause of cancer-related mortality in males worldwide [1]. The incidence of $\mathrm{PCa}$ is lower in Asian countries compared with European countries. However, given the changes in lifestyle and the declining proportion of the aging population in China, $\mathrm{PCa}$ increases faster than other malignant tumors [2]. Currently, prostate -specific antigen (PSA) is widely used as a diagnostic PCa molecular markers in clinical practice. However, due to the impact of benign prostatic hyperplasia, inflammation, age, drug, and other related factors, the specificity and sensitivity of PSA remains controversial $[3,4]$. An accurate marker for PCa diagnosis may help doctors and patients implement treatment. Many researchers have been looking for other highly specific diagnosis marker for PCa.

Epigenetic mechanisms that may be involved in the development of PCa have attracted considerable research interest $[5,6]$. Epigenetics refers to a DNA sequence that does not change. However, the gene undergoes a heritable change that alters the cell genetic material, and this change can be steadily transferred to cell proliferation. The epigenetic molecular mechanisms include DNA methylation, RNA interference, histone modifications, and chromatin modifications [7], among which DNA methylation is the most common. Previous studies have shown that aberrant DNA methylation is closely related to the development of tumors, such as that in $\mathrm{PCa}$ [8], bladder cancer [9], hepatocellular carcinoma [10], nasopharyngeal carcinoma [11], lung neoplasms [12], and gynecologic oncology [13]. Abnormal DNA methylation may occur from gene to site. A variety of cancer-related genes can be expressed in gene promoter methylation, activating the proliferation of tumor cell [5]. However, the diagnosis of $\mathrm{PCa}$ using $\mathrm{CpG}$-based biomarkers has been rarely reported.

To explore the relationships between the methylation levels of $\mathrm{CpG}$ sites and $\mathrm{PCa}$, we analyzed the differentially methylated sites of 423 primary $\mathrm{PCa}$ and 39 adjacent samples from The Cancer Genome Atlas (TCGA) database in this study. First, we utilized bioinformatics methods to mine eight diagnostic methylation sites $(\operatorname{cg} 06363129$, $\operatorname{cg} 07220448$, and $\operatorname{cg} 11417025$ in SOSTDC1; $\operatorname{cg} 08843517$ in CYBA; $\operatorname{cg} 03576469$ in CCDC8; $\operatorname{cg} 05385513$ in EFEMP1; cg20883831in KCNH2; and $\operatorname{cg} 23824801$ in CBX5) from TCGA. These sites are tissue specific and have high diagnostic efficacy that could distinguish $\mathrm{PCa}$ from adjacent samples. The result was further verified using the Gene Expression Omnibus (GEO) dataset. Finally, we tested five methylated sites in the gene promoter $(\operatorname{cg} 06363129, \operatorname{cg} 08843517, \operatorname{cg} 05385513$, $\operatorname{cg} 07220448$, and $\operatorname{cg} 11417025)$ through pyrosequencing in our samples. The combined diagnosis of four diagnostic methylation sites (cg06363129, cg08843517, cg05385513, and $\operatorname{cg} 11417025)$ showed high diagnostic efficacy and could be used in clinical research. These results may provide a novel potential biomarker for diagnosis of PCa.

\section{RESULTS}

\section{Differentially methylated CpG sites between PCa and adjacent samples from TCGA}

To explore the DNA methylome in PCa, we collected 423 primary $\mathrm{PCa}$ and 39 adjacent samples that were profiled through Illumina HumanMethylation450 platform from the TCGA (Table 1). The Illumina HumanMethylation450 array included 482,421 CpG sites. After quality filtering, 445,802 detected sites were analyzed. We used the limma software package for linear regression model analysis. Finally, a total of 15,744 differentially methylated CpGs (False Discovery Rate $<1$ E-10, |Delta Beta $\mid>0.2$ ) were found between PCa and adjacent samples. After removing the $\mathrm{CpGs}$ located in microRNA, 1,585 CpGs in the gene promoters were assayed in this study (Supplementary Table 2). Among these sites, we found that 139 hypermethylated and 1,446 hypomethylated CpGs were present in $\mathrm{PCa}$ (Figure 1A). Additionally, we observed that the differentially methylated sites were mainly located in CpG islands (44.79\%) and TSS200 region $(84.13 \%)$ in the gene promoter (Figure 1B and 1C).

To further explore the differentially methylated sites between tumor tissues and adjacent samples, we carried out gene pathway enrichment analysis for the matched genes. The hypermethylated sites were mainly involved in two pathways of olfactory transduction $(\mathrm{P}$-value $=5.33 \mathrm{E}-10)$ and ribosome $(\mathrm{P}$-value $=3.03 \mathrm{E}$ 04). The hypomethylated sites were mainly involved in 21 pathways, including neuroactive ligand-receptor interaction, cardiomyopathy, ras/hippo signaling pathway, and pathway in cancer (as shown in Figure 2D).

\section{Diagnostic methylation CpGs in PCa}

To investigate the diagnostic methylation markers in PCa from TCGA, we used the Prediction Analysis of Microarrays (PAM) package in Bioconductor to predict significant CpGs. After 10-fold cross-validation, we harvested eight diagnostic methylation CpGs (cg06363129, cg08843517, cg03576469, cg05385513, cg07220448, cg11417025, cg20883831, and cg23824801) in the gene promoter that were hypermethylation in PCa. Details were shown in Figure 3 and Supplementary Table 3. The Logistic regression model and the receiver operating characteristic (ROC) curves were used to evaluate the diagnostic efficiency of single site and combined diagnosis (Table 2 and Figure 2A). The area under curve (AUC) of ROC of a single site ranged from 0.910 to 0.939 , and the combined diagnosis of eight sites had a higher AUC of 0.942 (95\% confidence interval $=0.894-0.990, \mathrm{P}<0.001)$ than the single site. These methylation $\mathrm{CpGs}$ had a high diagnostic and could distinguish tumor tissues from adjacent tissues. 
Table 1: Clinical characteristics of prostate cancer patients from TCGA

\begin{tabular}{lcc}
\hline Variables & Prostate cancer $(\mathbf{n}=\mathbf{4 2 3})$ & Adjacent $(\mathbf{n}=\mathbf{3 9})$ \\
\hline Age(years) & $61(56,66)$ & $61(55,66)$ \\
Race & 136 & 38 \\
White & 7 & 1 \\
Black or African American & 2 & 0 \\
Asian & 278 & 0 \\
NA & & \\
Tumor size & 0 & 0 \\
T1 & 183 & 18 \\
T2 & 230 & 18 \\
T3 & 8 & 3 \\
T4 & 2 & 0 \\
NA & & 31 \\
Lymph node & 299 & 2 \\
N0 & 57 & 6 \\
N1 & 67 & \\
NA & & 0 \\
Metastasis status & 0 & 0 \\
M0 & 0 & 39 \\
M1 & 423 & \\
NA & & \\
\hline
\end{tabular}

NA: not available. The ages were presented as the median $(25 \%-75 \%$ quartiles $)$.
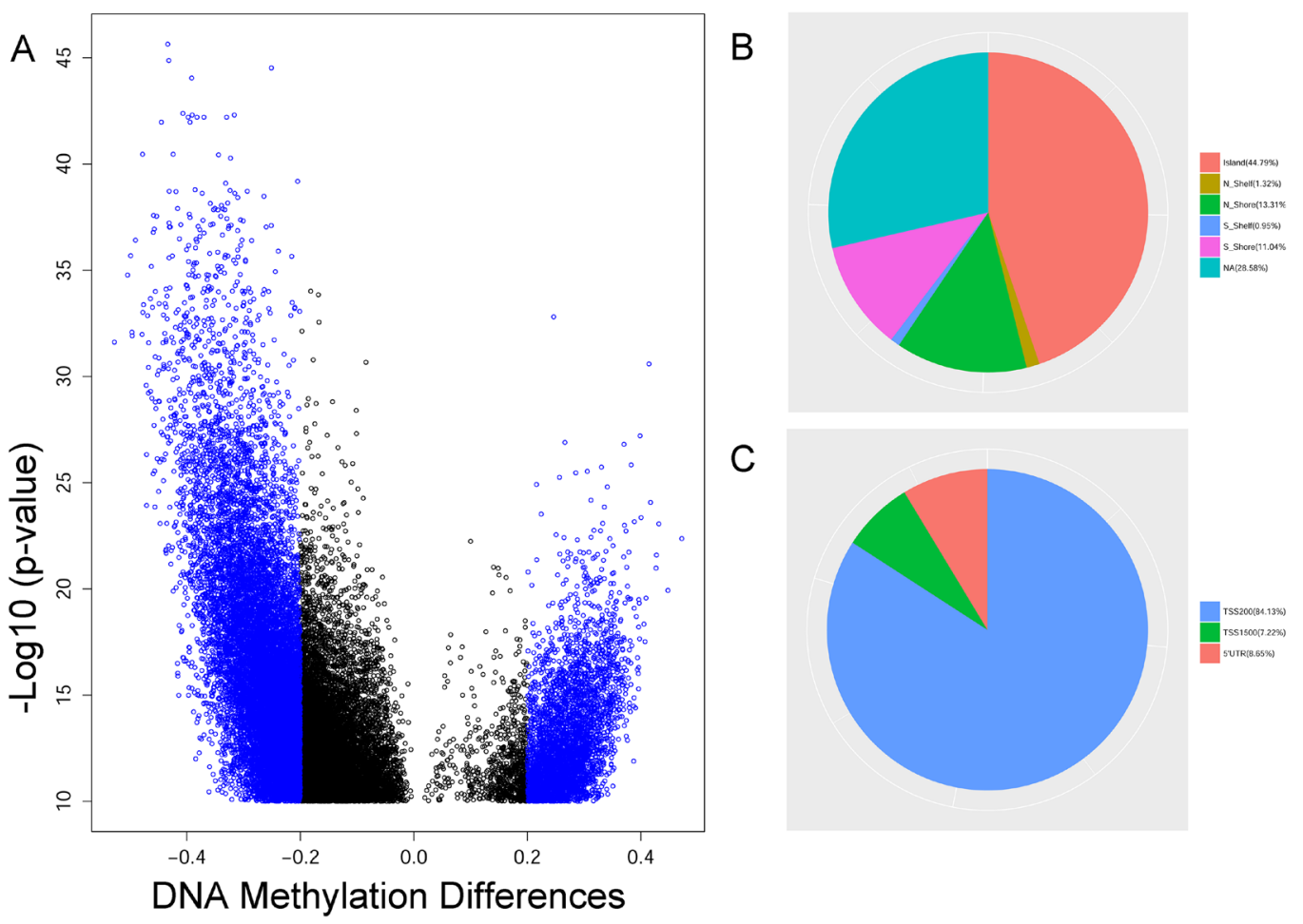

Figure 1: The analysis of differentially methylated sites from TCGA. (A) The volcano plot of differentially methylated sites with FDR $<1 \mathrm{E}-10$ between the tumor and the adjacent in prostate cancer. (B) The distribution of differentially methylated sites in promoter region. (C) The distribution of differentially methylated sites in $\mathrm{CpG}$ island. 


\section{Tissue specificity of diagnostic methylation CpGs in other solid tumors}

To further validate the tissue specificity of eight diagnostic methylation $\mathrm{CpGs}$ in $\mathrm{PCa}$, the datasets of other solid tumors from TCGA were analyzed. After removing the datasets that did not provide for the raw data detected by Illumina HumanMethylation450 platform, with less than 10 samples in tumors and adjacent tissues, or after neoadjuvant therapy was administered, we brought in a total of 11 solid tumors including $\mathrm{PCa}$ in the study (as shown in Supplementary Table 4). With $\mathrm{P}<0.05$ and $\mid$ Delta Beta| $>0.2$ in $\mathrm{PCa}$, we found that expect for $\mathrm{PCa}$, no more than two sites of these common hypermethylation of eight diagnostic methylation CpGs (cg06363129, cg08843517, $\operatorname{cg} 03576469, \operatorname{cg} 05385513, \operatorname{cg} 07220448, \operatorname{cg} 11417025$,
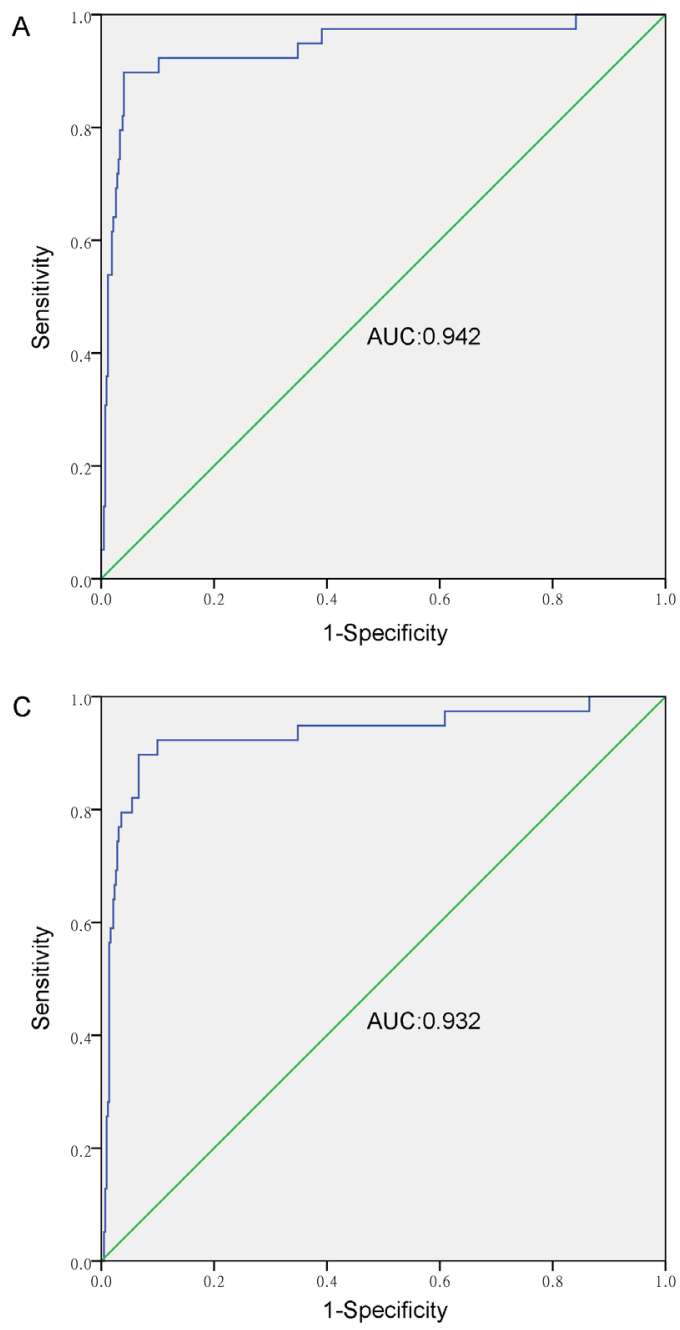

cg20883831, and $\operatorname{cg} 23824801)$ were shown in the other 10 solid tumors (bladder urothelial carcinoma, breast invasive carcinoma, colon adenocarcinoma, esophageal carcinoma, head and neck squamous cell carcinoma, kidney renal papillary cell carcinoma, liver hepatocellular carcinoma, lung adenocarcinoma, thyroid carcinoma, and uterine corpus endometrial carcinoma). A combination of theses hypermethylation CpGs had tissue specificity in $\mathrm{PCa}$ and may provide a novel potential biomarker for differentiating $\mathrm{PCa}$ from other tumors.

\section{Association of diagnostic methylation CpGs with Gleason Score (GS) in PCa tumors}

To explore the potential roles of these eight diagnostic methylation $\mathrm{CpGs}$ in the biological behavior or prognosis

B

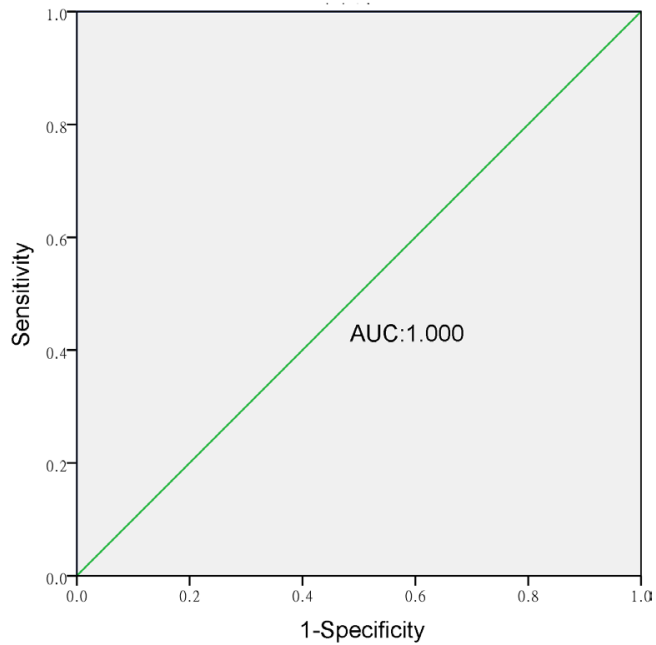

D

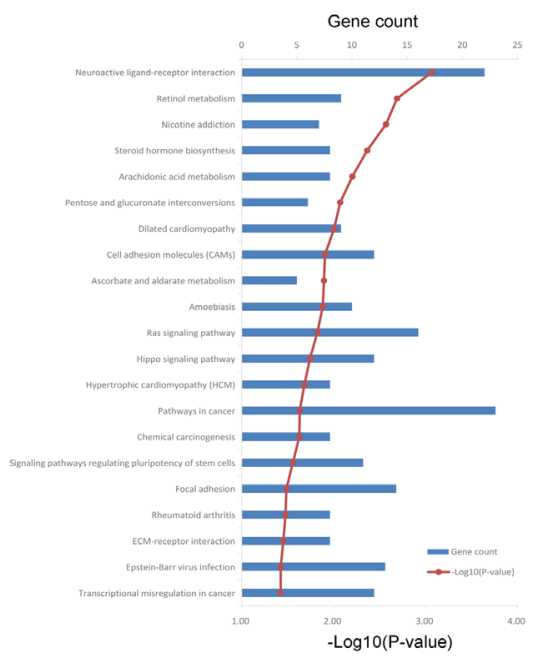

Figure 2: The analysis of diagnostic efficacy and gene pathway enrichment. (A) The ROC curve of a combined diagnosis of eight CpGs (cg06363129, cg08843517, cg03576469, cg05385513, cg07220448, cg11417025 and cg20883831) in gene promoter in 423 tumor samples and 39 adjacent tissues from TCGA. (B) The ROC curve of a combined diagnosis of eight CpGs (cg06363129, cg08843517, cg03576469, cg05385513, cg07220448, cg11417025 and cg20883831, and cg23824801) in gene promoter in 12 tumor samples and matched adjacent tissues from GSE74013. (C) The ROC curve of a combined diagnosis of four CpGs (cg06363129, cg08843517, cg05385513 and cg11417025) in gene promoter in 423 tumor samples and 39 adjacent tissues from TCGA. (D) Gene pathway enrichment analysis for differentially hypomethylated sites between 423 tumor tissues and 39 adjacent samples in PCa. 
of PCa, we assessed the methylation levels of these eight sites with different GS (Table 3). In this study, our samples included 1 cases GS6 $(2+4), 43$ cases GS6 $(3+3), 136$ cases GS7 $(3+4), 93$ cases GS7 $(4+3), 8$ cases GS8 $(3+$ 5), 37 cases GS8 $(4+4), 53$ cases GS $8(5+3), 68$ cases GS 9 $(4+5), 29$ cases GS9 $(5+4)$, and 1 cases GS10 $(5+5)$. We classified the PCa with GS into three groups (GS $\leq 6, \mathrm{GS}=$ 7 , and GS $\geq 8$ ) according to the results of a previous study [5]. After using the Kruskal-Wallis test among these three groups, we found that only the $\operatorname{cg} 06363129$ site showed a significant difference between the groups of GS $=7$ and GS $\geq 8(\mathrm{P}<0.05)$. The high Gleason grade in $\mathrm{PCa}$ had increased methylation levels of cg06363129, thereby suggesting that the methylation of $\operatorname{cg} 06363129$ may stimulate the progression of PCa.

\section{Correlations of diagnostic methylation CpGs and mRNA expression}

The scatter plots were carried out to describe the correlations between eight methylation CpGs and the corresponding gene mRNA expressions from TCGA (Figure
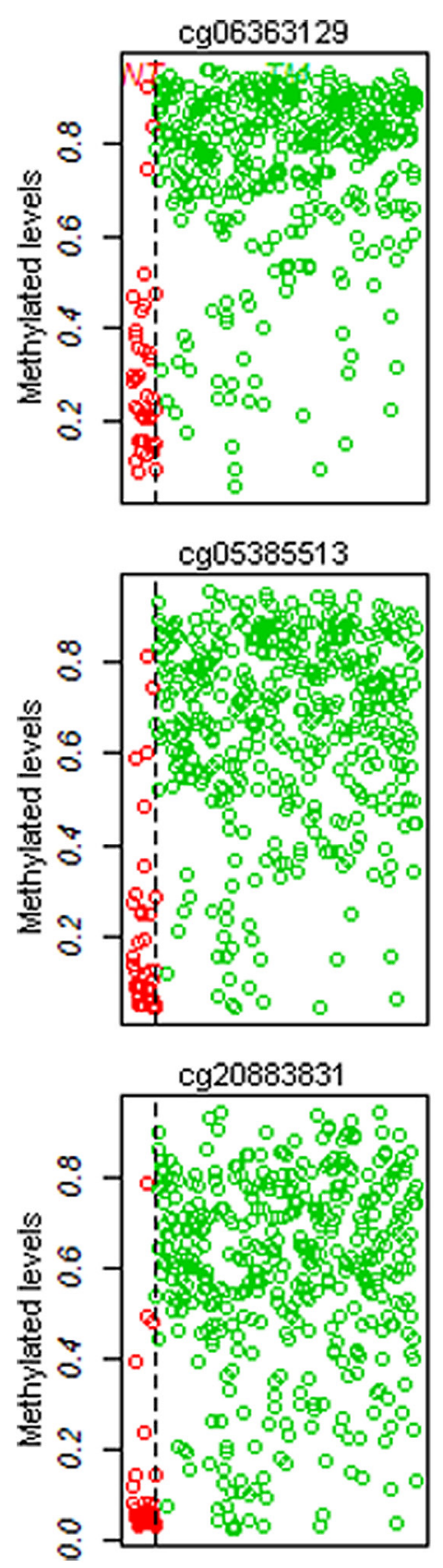
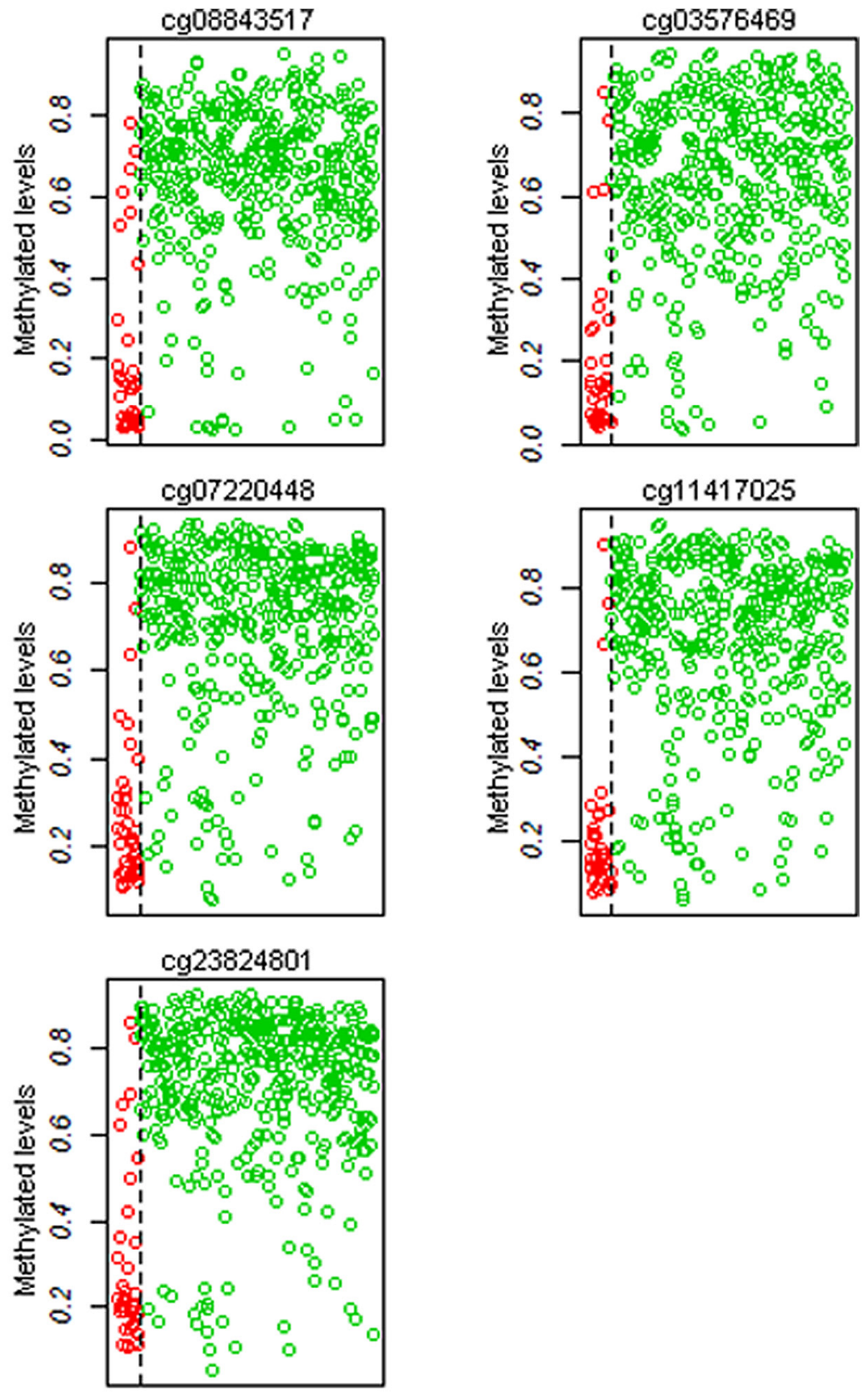

Figure 3: The methylation levels of eight CpGs (cg06363129, cg08843517, cg03576469, cg05385513, cg07220448, cg11417025 and cg20883831, and cg23824801) in 423 tumor samples and 39 adjacent tissues from TCGA. 
Table 2: Diagnostic efficiency of single site

\begin{tabular}{lcccc}
\hline Variables & AUC & Standard error & P- value & 95\% CI \\
\hline $\operatorname{cg} 06363129$ & 0.922 & 0.028 & 0.000 & $0.867-0.977$ \\
$\operatorname{cg} 08843517$ & 0.913 & 0.027 & 0.000 & $0.860-0.966$ \\
$\operatorname{cg} 03576469$ & 0.919 & 0.029 & 0.000 & $0.861-0.976$ \\
$\operatorname{cg} 05385513$ & 0.927 & 0.024 & 0.000 & $0.879-0.975$ \\
$\operatorname{cg} 07220448$ & 0.927 & 0.025 & 0.000 & $0.878-0.977$ \\
$\operatorname{cg} 11417025$ & 0.923 & 0.029 & 0.000 & $0.867-0.979$ \\
$\operatorname{cg} 20883831$ & 0.939 & 0.023 & 0.000 & $0.894-0.983$ \\
$\operatorname{cg} 23824801$ & 0.910 & 0.028 & 0.000 & $0.855-0.965$ \\
\hline
\end{tabular}

AUC: area under roc curve; 95\% CI: 95\% confidence interval, P- value $<0.05$ indicated a significant difference.

Table 3: Association of site methylation levels with Gleason score in PCa tumors

\begin{tabular}{lccc}
\hline \multirow{2}{*}{ Variables } & $\mathbf{G S} \leq \mathbf{6}$ & $\mathbf{G S}=\mathbf{7}$ & $\mathbf{G S} \geq \mathbf{8}$ \\
\cline { 2 - 4 } & $\mathbf{( n = 4 4 )}$ & $\mathbf{( n = 2 2 9 )}$ & $\mathbf{( n = 1 5 0 )}$ \\
\hline $\operatorname{cg} 06363129$ & $0.825(0.753-0.886)$ & $0.800(0.693-0.876)^{\mathrm{a}}$ & $0.845(0.737-0.901)^{\mathrm{a}}$ \\
$\operatorname{cg} 08843517$ & $0.659(0.619-0.773)$ & $0.681(0.561-0.767)$ & $0.695(0.581-0.788)$ \\
$\operatorname{cg} 03576469$ & $0.674(0.549-0.790)$ & $0.679(0.526-0.792)$ & $0.721(0.578-0.837)$ \\
$\operatorname{cg} 05385513$ & $0.669(0.572-0.815)$ & $0.696(0.545-0.818)$ & $0.728(0.580-0.844)$ \\
$\operatorname{cg} 07220448$ & $0.759(0.701-0.827)$ & $0.755(0.629-0.836)$ & $0.802(0.663-0.857)$ \\
$\operatorname{cg} 11417025$ & $0.728(0.635-0.817)$ & $0.705(0.559-0.810)$ & $0.757(0.600-0.842)$ \\
$\operatorname{cg} 20883831$ & $0.631(0.461-0.764)$ & $0.602(0.474-0.730)$ & $0.659(0.495-0.779)$ \\
$\operatorname{cg} 23824801$ & $0.767(0.685-0.838)$ & $0.764(0.652-0.841)$ & $0.779(0.660-0.842)$ \\
\hline
\end{tabular}

GS: Gleason score. Data were provided as median ( $25 \%-75 \%$ quartiles). The Kruskal-Wallis test was conducted among three groups. P-value $<0.05$ indicated a significant difference.

a: At the site of $\operatorname{cg} 06363129$, the groups of $\mathrm{GS}=7$ and $\mathrm{GS} \geq 8$ had a significant difference.

4). We observed that the correlations between $\operatorname{cg} 06363129$, $\operatorname{cg} 07220448$, or $\operatorname{cg} 11417025$ and SOSTDC1 expression in patients were all significant $(\mathrm{P}<0.001)$. The same results were also found between $\operatorname{cg} 08843517$ and CYBA, $\operatorname{cg} 03576469$ and CCDC8, and $\operatorname{cg} 05385513$ and EFEMP1, in which the correlation coefficient reached 0.5 , expect for cg20883831in KCNH2. However, all P-value were less than 0.001 . No correlations between $\operatorname{cg} 23824801$ and CBX5 mRNA expression $(r=-0.042, P=0.377)$ were noted.

\section{Verification of eight methylation sites in GEO dataset}

To further verify the methylation levels of sites in PCa, the GSE74013 dataset was analyzed in the study. The diagnostic sites $(\operatorname{cg} 06363129, \operatorname{cg} 08843517, \operatorname{cg} 03576469$, $\operatorname{cg} 05385513, \operatorname{cg} 07220448, \operatorname{cg} 11417025, \operatorname{cg} 20883831$, and cg23824801) showed significantly higher methylation in $\mathrm{PCa}$ than that in the adjacent tissues $(\mathrm{P}<0.01)$ (Supplementary Figure 1). At the same time, an AUC of $1.000(95 \% \mathrm{CI}=1.000-1.000, \mathrm{P}<0.001)$ was found for the combined diagnosis of eight sites on GEO dataset (Figure 2B). These results supported the data from TCGA.

\section{Detection of methylation sites through pyrosequencing}

To further improve the application value of the combined diagnosis, we excluded the lowest tissuespecific site of cg03576469 that was hypermethylated in several solid cancers, such as breast invasive carcinoma, colon adenocarcinoma, esophageal carcinoma, and lung adenocarcinoma. Additionally, cg20883831 in KCNH2 and cg23824801 in CBX5 were also excluded due to low correlation coefficient $(\mathrm{r}<0.5)$. At last, we verified five methylated sites ( $\operatorname{cg} 06363129, \operatorname{cg} 07220448, \operatorname{cg} 11417025$ 
in SOSTDC1; $\operatorname{cg} 08843517$ in CYBA; and $\operatorname{cg} 05385513$ in EFEMP1) in PCa and normal tissues, including adjacent tissues and benign prostatic hyperplasia $(\mathrm{BPH})$ through pyrosequencing (Figure 5). The methylation levels of cg06363129, cg08843517, $\operatorname{cg} 05385513$, and $\operatorname{cg} 11417025$ were higher in $\mathrm{PCa}$ than that in the normal tissues $(\mathrm{P}<$ 0.05). Compared with normal tissues, the methylation levels of $\operatorname{cg} 07220448$ had no statistical differences in $\mathrm{PCa}$ $(\mathrm{P}=0.0631)$. These findings further confirmed the results of TCGA and GEO from experimental data.
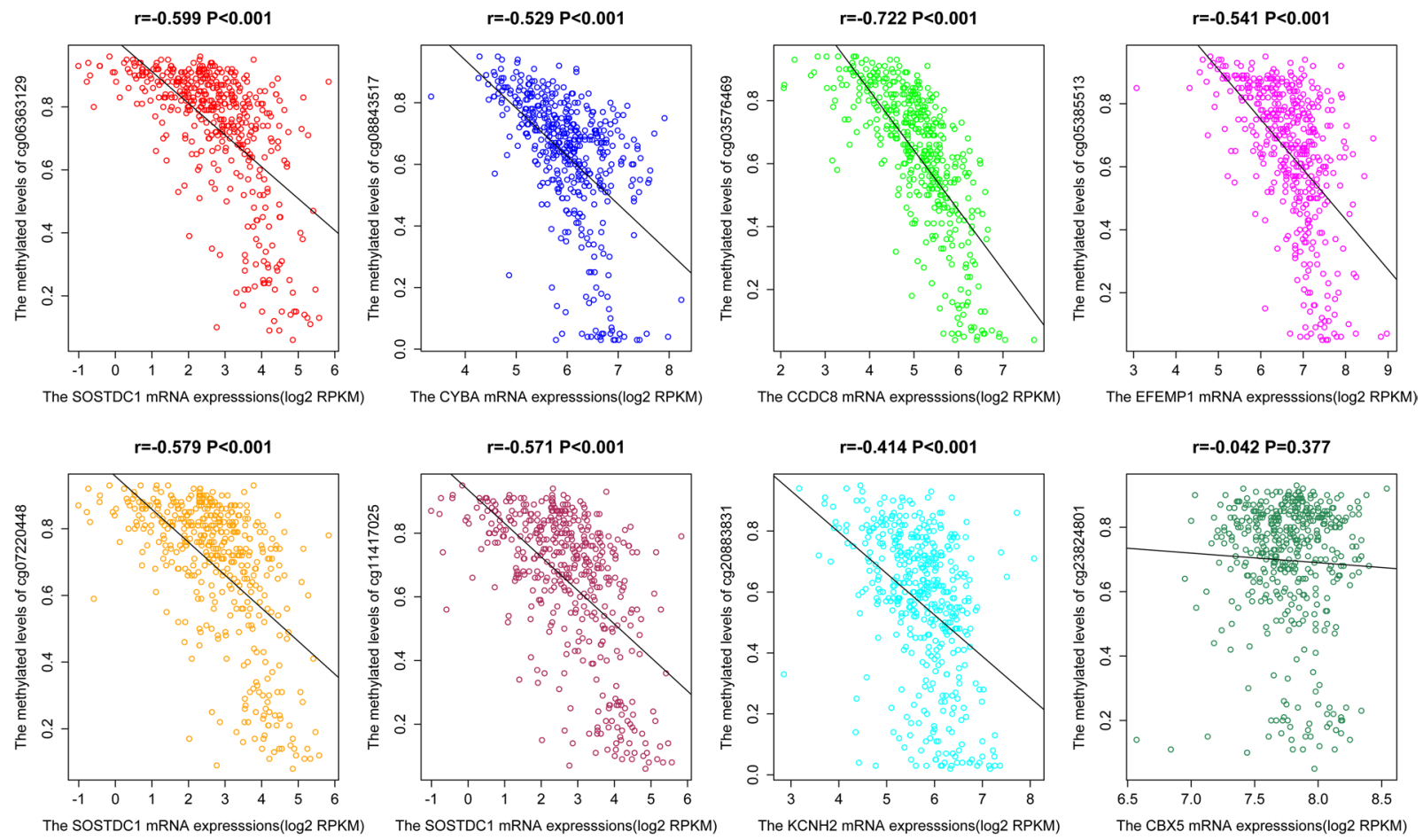

Figure 4: The scatter plot of the correlation of eight CpGs (cg06363129, cg08843517, cg03576469, cg05385513, cg07220448, cg11417025 and cg20883831, and cg23824801) and gene mRNA expression in PCa from TCGA.
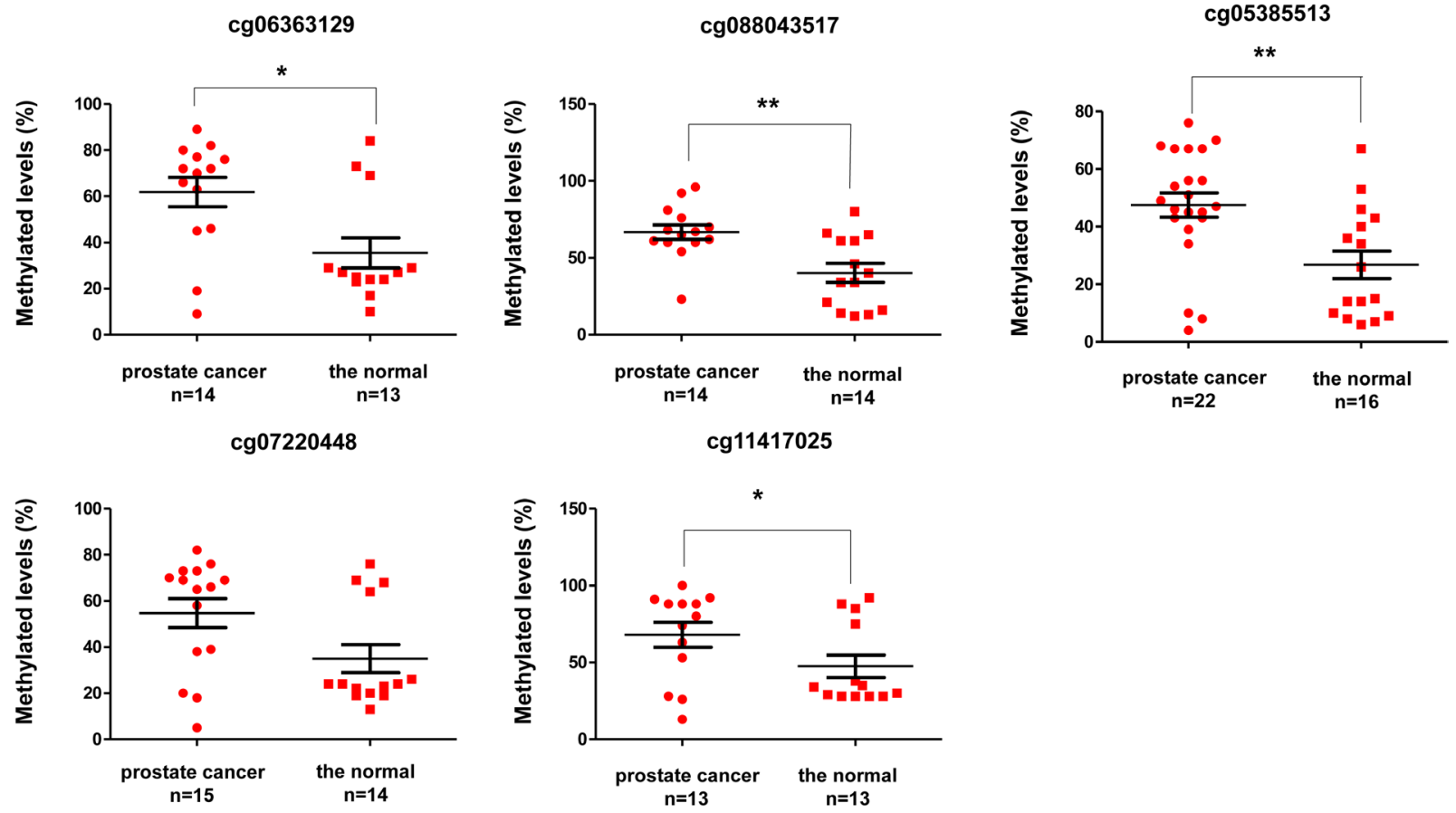

Figure 5: Methylation levels between prostate cancer and normal tissues through pyrosequencing. 
Table 4: Basic characteristics of prostate cancer samples used in pyrosequencing analysis

\begin{tabular}{|c|c|c|}
\hline Variables & Prostate cancer $(n=22)$ & The normal $(n=18)$ \\
\hline Age(years) & $68.00(65.50,72.25)$ & $64.00(67.00,71.25)$ \\
\hline \multicolumn{3}{|l|}{ Category } \\
\hline Adjacent & - & 13 \\
\hline $\mathrm{BPH}$ & - & 5 \\
\hline \multicolumn{3}{|l|}{ Tumor size } \\
\hline $\mathrm{T} 1$ & 0 & 0 \\
\hline $\mathrm{T} 2$ & 8 & 8 \\
\hline $\mathrm{T} 3$ & 5 & 5 \\
\hline $\mathrm{T} 4$ & 0 & 0 \\
\hline NA & 9 & 5 \\
\hline \multicolumn{3}{|c|}{ Lymph node } \\
\hline No & 11 & 11 \\
\hline N1 & 1 & 1 \\
\hline NA & 10 & 6 \\
\hline \multicolumn{3}{|c|}{ Metastasis status } \\
\hline M0 & 12 & 12 \\
\hline M1 & 0 & 0 \\
\hline NA & 10 & 6 \\
\hline \multicolumn{3}{|c|}{ Gleason Score } \\
\hline $\mathrm{GS} \leq 6$ & 4 & 1 \\
\hline $\mathrm{GS}=7$ & 9 & 6 \\
\hline $\mathrm{GS} \geq 8$ & 8 & 6 \\
\hline NA & 1 & 5 \\
\hline
\end{tabular}

NA: not available. The ages were presented as the median ( $25 \%-75 \%$ quartiles).

Based on the results from TCGA, GEO and our experiment, we found that the combined diagnosis of four methylation sites (cg06363129, cg08843517, cg05385513, and cg11417025) had tissue specificity in PCa than other solid tumors (Supplementary Table 4), and a high diagnostic efficacy that reached up to 0.932 in TCGA (Figure 2C), 1.000 in GEO, and 0.899 in our samples, thereby indicating that these markers could be better used in clinical research.

\section{DISCUSSION}

DNA methylation occurring in the 5'-CG-3' gene sequence is considered as two nucleotides cytosine DNA methylation of CG being selectively added to form 5-methylcytosine. In tumorigenesis, the methylation model tends to be highly modified on $\mathrm{CpG}$ islands, resulting in reduction and inactivation of gene expression. In our study, we analyzed the differentially methylated sites of 423 primary PCas and 39 adjacent samples from TCGA database and found that DNA hypomethylation mainly occurs in $\mathrm{PCa}$, and differentially methylated sites are mainly located on $\mathrm{CpG}$ islands and TSS200 region in the gene promoter. Additionally, eight methylation sites (cg06363129, $\operatorname{cg} 07220448$, and $\operatorname{cg} 11417025$ in SOSTDC1; cg08843517 in CYBA; cg03576469 in CCDC8; cg05385513 in EFEMP1; cg20883831in KCNH2; and cg23824801in CBX5) with high tissue specificity and diagnostic efficacy were mined from TCGA, and the dataset from GEO further proved this result. Among them, the methylation levels of five CpG sites ( $\mathrm{cg} 06363129$, $\operatorname{cg} 08843517, \operatorname{cg} 03576469, \operatorname{cg} 07220448$, and $\operatorname{cg} 11417025)$ had negative correlations with gene expressions $(r>0.5$ and $\mathrm{P}<0.001)$ and the high methylation of $\operatorname{cg} 06363129$ may stimulate the progression of PCa. Pyrosequencing in our samples confirmed that the combined diagnosis of four methylation sites (cg06363129, cg08843517, cg05385513, and cg11417025) had high diagnostic efficacy in PCa and could be better used in clinical research.

Sclerostin domain containing protein 1(SOSTDC1) is a member of the sclerostin family. SOSTDC1was reported to be involved in the $\mathrm{Wnt} / \mathrm{bone}$ morphogenetic protein signaling in bone metabolism [14]. In addition, it also suppresses tumorigenesis. Results of TCGA and GEO dataset showed that the diagnostic methylation 
CpGs (cg06363129, cg07220448, and cg11417025) in PCa located at promoter region of SOSTDC1 and the methylation levels of sites had negative correlations with SOSTDC1 mRNA expression. SOSTDC1 regulates the expressions of p21Cip and p27Kip to inhibit the proliferation of non-small-cell lung cancer cells [15]. SOSTDC1 inhibits cell proliferation and differentiation and induces G1/S arrest in thyroid cancer [16]. In prostate cells, researchers have found that SOSTDC1 regulates the levels of hepcidin by inhibiting BMP and Wnt signaling, and the promoter methylation of SOSTDC1 is associated with tumor recurrence in $\mathrm{PCa}$ [17]. These results suggest that the high methylation model at cg06363129 or cg11417025 may decrease SOSTDC1 expression, promoting PCa development. Gleason Score, which is associated with the biological behavior and prognosis, has been recognized as an important reference index in PCa. In our study, high Gleason grade in PCa had greater methylation levels of cg06363129, suggesting that high methylation of $\operatorname{cg} 06363129$ may stimulate the progression of PCa.

Similar results were also found for $\operatorname{cg} 08843517$ located in cytochrome b light chain (CYBA). CYBA, which is encoded by a polymorphic gene, is involved in the process of electron transport and superoxide anion production [18]. CYBA has been reported to be associated with melanoma through DNA hypermethylation during tumor progression [19]. CYBA also displays increased methylation in several invasive cell lines in malignant melanoma [20]. In prostate cancer cells, androgens can induce oxidative stress and radiation resistance by increasing the expressions of $\mathrm{p} 22^{\text {phox }}$ and gp91 ${ }^{\text {phox }}$ [21]. CYBA is also involved in the biological processes of angiogenesis and tumor growth by regulating AKT and ERK1/2 signaling pathways [22]. In our study, the high methylation of $\operatorname{cg} 08843517$ is related to CYBA mRNA expression, and this may provide a novel idea for exploring the mechanism of $\mathrm{PCa}$.

The EGF-containing fibulin-like extracellular matrix protein 1 (EFEMP1), an extracellular matrix (ECM) protein, is associated with the tumorigenesis in different types of carcinoma [23]. In our study, the diagnostic methylation of $\operatorname{cg} 05385513$, which is located at the promoter region of EFEMP1, had higher methylation level in tumor than that in the normal tissues. From the tissue level, high methylation in EFEMP1 promoter may down-regulate mRNA expression, leading to cell proliferation [24]. From the cell level, by studying PCa cell lines, EFEMP1 mRNA expression was confirmed to be significantly lower in PCa than in the normal tissues [25]. The $\operatorname{cg} 05385513$ may play an important role in the promoter region of EFEMP1 that regulates cell adhesion and proliferation [25].

In conclusion, we utilized bioinformatics method to mine the diagnosis methylation CpGs from TCGA, and we found that the combined diagnosis of four methylation CpGs (cg06363129, cg08843517, cg05385513, and cg11417025) have high tissue specificity and diagnosis efficacy in $\mathrm{PCa}$. The cg06363129 located in SOSTDC1 may be involved in the progression of PCa. These results revealed a novel potential biomarker for diagnosis of the $\mathrm{PCa}$.

\section{MATERIALS AND METHODS}

\section{Methylation analysis of TCGA data}

All data (clinical parameters, methylation levels, and gene expressions) of PCa and adjacent tissues were obtained from TCGA up to October 10, 2015. The adjacent tissues were defined as a distance that was greater than $2 \mathrm{~cm}$ from tumor margin. All patients with a history of other malignancies or those who received neoadjuvant therapy (discrepancy) or radiation therapy were excluded in this study. The methylation values, which came from Illumina HumanMethylation450 BeadChips, were obtained from TCGA using RnBeads software (version 0.99) as previous [26]. The batch effects and surrogate variables were removed with the Surrogate Variable Analysis (SVA) package in R (http://www.bioconductor.org) [27]. The diagnostic methylation markers in $\mathrm{PCa}$ were explored using the PAM package in Bioconductor, and the gene enrichment analysis was carried out on David Bioinformatics Resources (version 6.8, NIAID/NIH) (https://david-d. ncifcrf.gov). To further validate the diagnostic markers in $\mathrm{PCa}$, the diagnostic efficacy of the methylation CpGs was evaluated using the ROC curves, and the relationships between the tissue specificity the prognosis (PCa with GS), gene expression (level 3 data, RNA-seq Version 2), and the methylation levels of CpGs were analyzed.

\section{Patient samples}

A total of 13 paired PCa and adjacent tissues were collected through surgical resection in Hunan Province Tumor Hospital, and nine $\mathrm{PCa}$ and five $\mathrm{BPH}$ biopsy samples were obtained from the 303rd Hospital of Chinese People's Liberation Army, Guangxi, China from June 15, 2015 to April 30, 2016 with informed consent obtained from all individual participants (Table 4). All patients had primary PCa and without history of chemotherapy or radiotherapy. After surgical resection or biopsy, the fresh specimens were determined by a pathologist as previously described [5] and immediately stored at $-80^{\circ} \mathrm{C}$ until analysis. This study was approved by the Medical Ethics Committee of Guangxi Medical University.

\section{Validation of GEO dataset}

The methylation data of $\mathrm{PCa}$ on Illumina HumanMethylation450 array were searched on GEO database (http:/www.ncbi.nlm.nih.gov/geo/) by using keywords "(prostate carcinoma or prostate cancer) and (methylation)" up to June 12, 2016. The inclusion criteria for datasets in the study were (1) the datasets provided 
the raw data of PCa and adjacent tissues, (2) sample source was restricted to humans, (3) the sample was not involved in adjuvant therapy, and (4) the number of PCa and adjacent samples was more than 10. Finally, only one dataset (GSE74013) was included in our study, and this contained $12 \mathrm{PCa}$ and 12 matched adjacent samples. The methylation levels were calculated using $\beta$-value as previously described: $\beta=$ methylated intensity/(methylated intensity + unmethylated +100$)[11]$

\section{Quantitative pyrosequencing}

DNA was extracted with Genomic DNA Extraction Kit (TaKaRa, Dalian, China). A total of 500 ng DNA was bisulfite converted through EZ DNA Methylation-Gold $\mathrm{Kit}^{\mathrm{TM}}$ (D5005, Zymo Research, United States). We tested five methylation sites for validation through pyrosequencing. The specific primers were designed with Pyromark Assay Design software (version 2.0; Qiagen) (the primers were shown in Supplementary Table 1), and the methylation sites were included in the analysis sequence. Three different $\mathrm{CpG}$ island loci (cg06363129, cg07220448, cg11417025) were close to each other that located at the genomic position of 16505602, 16505592, and 16505589 in SOSTDC1 respectively in Supplementary Table 3. Therefore, they had the same primer that can measure the methylated levels of three loci simultaneously. The polymerase chain reaction (PCR) was carried out by using PyroMark PCR Kits (Qiagen). A total of $25 \mu$ volume was amplified with $50 \mathrm{ng}$ bisulfite-converted DNA, $1 \times$ Pyromark PCR master Mix 12.5 $\mu \mathrm{l}, 1 \times$ CoralLoad Concentration $2.5 \mu \mathrm{l}$, 8ul RNase-free water, $0.5 \mathrm{ul} 0.2 \mathrm{Um}$ forward, and $0.5 \mu \mathrm{l} 0.2 \mathrm{Um} 5$ 'biotinylatedreverse primer. Thermal cycling conditions included an initial denaturation at $95{ }^{\circ} \mathrm{C}$ for $15 \mathrm{~min}$, followed by 45 cycles of denaturation $\left(30 \mathrm{~s}\right.$ at $\left.94^{\circ} \mathrm{C}\right)$, annealing $(30 \mathrm{~s}$ at 56 ${ }^{\circ} \mathrm{C}$ ), and extension $\left(30 \mathrm{~s}\right.$ at $\left.72^{\circ} \mathrm{C}\right)$, and then final extension at $72{ }^{\circ} \mathrm{C}$ for $10 \mathrm{~min}$ and cooling to $4{ }^{\circ} \mathrm{C}$ until analysis. Pyrosequencing was carried out to detect the methylation levels of CpG sites through PyroMark Q96 ID Software (Qiagen) according to the manufacturer's instruction.

\section{Statistical analysis}

The methylation levels of CpGs were presented as the median $(25 \%-75 \%$ quartiles $)$. The differences in the tumor and the normal tissues were provided as Delta Beta. The Mann-Whitney U or the Wilcoxon's matched pairs test was conducted between two groups, and the KruskalWallis test was used among three groups. The associations between methylation sites and gene mRNA expression were assessed through Pearson's rank-correlation coefficients. The logistic regression model and an AUC of ROC curves with $95 \%$ CI were used to predict diagnostic efficacy of the combination of methylation sites as previously described [28]. Statistical analysis was performed with IBM SPSS (Version 20.0, Inc., Chicago, IL, USA) and GraphPad Prism (Version 5.0, GraphPad Software; San Diego, CA,
USA). The statistical tests were two-tailed, and $P<0.05$ was regarded as statistically significant.

\section{ACKNOWLEDGMENTS}

This research was supported in part by grants from the National Natural Science Foundation of China (\#81272853, \#81472414, and \#81370857), Guangxi scientific research and technology development project (Guikegong 2013BC26299 and 1355005-3$17)$, and the Guangxi Natural Science Foundation (2015GXNSFBB139008, 2014GXNSFBA118201, and 2013GXNSFFA019002).

\section{CONFLICTS OF INTEREST}

The authors state no conflicts of interest.

\section{REFERENCES}

1. Siegel R, Ma J, Zou Z, Jemal A. Cancer statistics, 2014. CA Cancer J Clin. 2014; 64:9-29. doi: 10.3322/caac.21208.

2. Lughezzani G, Lazzeri M, Haese A, McNicholas T, de la Taille A, Buffi NM, Fossati N, Lista G, Larcher A, Abrate A, Mistretta A, Bini V, Palou Redorta J, et al. Multicenter European external validation of a prostate health indexbased nomogram for predicting prostate cancer at extended biopsy. Eur Urol. 2014; 66:906-12. doi: 10.1016/j. eururo.2013.12.005.

3. Castillejos-Molina RA, Gabilondo-Navarro FB. Prostate cancer. Salud Publica Mex. 2016; 58:279-84.

4. Lazzeri M, Haese A, de la Taille A, Palou Redorta J, McNicholas T, Lughezzani G, Scattoni V, Bini V, Freschi M, Sussman A, Ghaleh B, Le Corvoisier P, Alberola Bou J, et al. Serum isoform [-2]proPSA derivatives significantly improve prediction of prostate cancer at initial biopsy in a total PSA range of 2-10 ng/ml: a multicentric European study. Eur Urol. 2013; 63:986-94. doi: 10.1016/j. eururo.2013.01.011.

5. Kobayashi Y, Absher DM, Gulzar ZG, Young SR, McKenney JK, Peehl DM, Brooks JD, Myers RM, Sherlock G. DNA methylation profiling reveals novel biomarkers and important roles for DNA methyltransferases in prostate cancer. Genome Res. 2011; 21:1017-27. doi: 10.1101/ gr.119487.110.

6. White-Al Habeeb NM, Ho LT, Olkhov-Mitsel E, Kron K, Pethe V, Lehman M, Jovanovic L, Fleshner N, van der Kwast T, Nelson CC, Bapat B. Integrated analysis of epigenomic and genomic changes by DNA methylation dependent mechanisms provides potential novel biomarkers for prostate cancer. Oncotarget. 2014; 5:7858-69. doi: 10.18632/oncotarget.2313.

7. Piletic K, Kunej T. MicroRNA epigenetic signatures in human disease. Arch Toxicol. 2016; 90:2405-19. doi: 10.1007/s00204-016-1815-7. 
8. Damaschke NA, Yang B, Blute ML Jr, Lin CP, Huang W, Jarrard DF. Frequent disruption of chromodomain helicase DNA-binding protein 8 (CHD8) and functionally associated chromatin regulators in prostate cancer. Neoplasia. 2014; 16:1018-27. doi: 10.1016/j.neo.2014.10.003.

9. Kim YW, Yoon HY, Seo SP, Lee SK, Kang HW, Kim WT, Bang HJ, Ryu DH, Yun SJ, Lee SC, Kim WJ, Kim YJ. Clinical implications and prognostic values of prostate cancer susceptibility candidate methylation in primary nonmuscle invasive bladder cancer. Dis Markers. 2015; 2015:402963. doi: 10.1155/2015/402963.

10. Shen J, Wang S, Zhang YJ, Kappil M, Wu HC, Kibriya MG, Wang Q, Jasmine F, Ahsan H, Lee PH, Yu MW, Chen CJ, Santella RM. Genome-wide DNA methylation profiles in hepatocellular carcinoma. Hepatology. 2012; 55:1799-808. doi: 10.1002/hep.25569.

11. Dai W, Cheung AK, Ko JM, Cheng Y, Zheng H, Ngan RK, Ng WT, Lee AW, Yau CC, Lee VH, Lung ML. Comparative methylome analysis in solid tumors reveals aberrant methylation at chromosome $6 \mathrm{p}$ in nasopharyngeal carcinoma. Cancer Med. 2015; 4:1079-90. doi: 10.1002/ cam4.451.

12. Freeman JR, Chu S, Hsu T, Huang YT. Epigenome-wide association study of smoking and DNA methylation in nonsmall cell lung neoplasms. Oncotarget. 2016; 7:69579-91. doi: 10.18632/oncotarget.11831.

13. Klinkebiel D, Zhang W, Akers SN, Odunsi K, Karpf AR. DNA methylome analyses implicate fallopian tube epithelia as the origin for high-grade serous ovarian cancer. Mol Cancer Res. 2016; 14:787-94. doi: 10.1158/1541-7786. mor-16-0097.

14. Collette NM, Yee CS, Hum NR, Murugesh DK, Christiansen BA, Xie L, Economides AN, Manilay JO, Robling AG, Loots GG. Sostdc1 deficiency accelerates fracture healing by promoting the expansion of periosteal mesenchymal stem cells. Bone. 2016; 88:20-30. doi: 10.1016/j.bone.2016.04.005.

15. Liu L, Wu S, Yang Y, Cai J, Zhu X, Wu J, Li M, Guan H. SOSTDC1 is down-regulated in non-small cell lung cancer and contributes to cancer cell proliferation. Cell Biosci. 2016; 6:24. doi: 10.1186/s13578-016-0091-9.

16. Liang W, Guan H, He X, Ke W, Xu L, Liu L, Xiao H, Li Y. Down-regulation of SOSTDC1 promotes thyroid cancer cell proliferation via regulating cyclin A2 and cyclin E2. Oncotarget. 2015; 6:31780-91. doi: 10.18632/ oncotarget.5566.

17. Tesfay L, Clausen KA, Kim JW, Hegde P, Wang X, Miller LD, Deng Z, Blanchette N, Arvedson T, Miranti CK, Babitt JL, Lin HY, Peehl DM, et al. Hepcidin regulation in prostate and its disruption in prostate cancer. Cancer Res. 2015; 75:2254-63. doi: 10.1158/0008-5472.can-14-2465.

18. Zhou H, Chen M, Zhu Y, Wang B, Liu XN, Zuo Z, Tang FY. Polymorphisms in NADPH oxidase CYBA gene modify the risk of ESRD in patients with chronic glomerulonephritis. Ren Fail. 2016; 38:262-7. doi: 10.3109/0886022x.2015.1117905.

19. Gallagher WM, Bergin OE, Rafferty M, Kelly ZD, Nolan IM, Fox EJ, Culhane AC, McArdle L, Fraga MF, Hughes L, Currid CA, O'Mahony F, Byrne A, et al. Multiple markers for melanoma progression regulated by DNA methylation: insights from transcriptomic studies. Carcinogenesis. 2005; 26:1856-67. doi: 10.1093/carcin/bgi152.

20. Faller WJ, Rafferty M, Hegarty S, Gremel G, Ryan D, Fraga MF, Esteller M, Dervan PA, Gallagher WM. Metallothionein 1E is methylated in malignant melanoma and increases sensitivity to cisplatin-induced apoptosis. Melanoma Res. 2010; 20:392-400.

21. Lu JP, Monardo L, Bryskin I, Hou ZF, Trachtenberg J, Wilson BC, Pinthus JH. Androgens induce oxidative stress and radiation resistance in prostate cancer cells though NADPH oxidase. Prostate Cancer Prostatic Dis. 2010; 13:39-46. doi: 10.1038/pcan.2009.24.

22. Li Q, Fu GB, Zheng JT, He J, Niu XB, Chen QD, Yin Y, Qian X, Xu Q, Wang M, Sun AF, Shu Y, Rui H, et al. NADPH oxidase subunit p22(phox)-mediated reactive oxygen species contribute to angiogenesis and tumor growth through AKT and ERK1/2 signaling pathways in prostate cancer. Biochim Biophys Acta. 2013; 1833:337585. doi: 10.1016/j.bbamcr.2013.09.018.

23. Yin X, Fang S, Wang M, Wang Q, Fang R, Chen J. EFEMP1 promotes ovarian cancer cell growth, invasion and metastasis via activated the AKT pathway. Oncotarget. 2016; 7:47938-53. doi: 10.18632/oncotarget.10296.

24. Almeida M, Costa VL, Costa NR, Ramalho-Carvalho J, Baptista T, Ribeiro FR, Paulo P, Teixeira MR, Oliveira J, Lothe RA, Lind GE, Henrique R, Jeronimo C. Epigenetic regulation of EFEMP1 in prostate cancer: biological relevance and clinical potential. J Cell Mol Med. 2014; 18:2287-97. doi: 10.1111/jcmm.12394.

25. Kim YJ, Yoon HY, Kim SK, Kim YW, Kim EJ, Kim IY, Kim WJ. EFEMP1 as a novel DNA methylation marker for prostate cancer: array-based DNA methylation and expression profiling. Clin Cancer Res. 2011; 17:4523-30. doi: 10.1158/1078-0432.ccr-10-2817.

26. Assenov Y, Muller F, Lutsik P, Walter J, Lengauer T, Bock C. Comprehensive analysis of DNA methylation data with RnBeads. Nat Methods. 2014; 11:1138-40. doi: 10.1038/ nmeth.3115.

27. Leek JT, Johnson WE, Parker HS, Jaffe AE, Storey JD. The sva package for removing batch effects and other unwanted variation in high-throughput experiments. Bioinformatics. 2012; 28:882-3. doi: 10.1093/bioinformatics/bts034.

28. Peng HX, Yang L, He BS, Pan YQ, Ying HQ, Sun HL, Lin $\mathrm{K}, \mathrm{Hu}$ XX, Xu T, Wang SK. Combination of preoperative NLR, PLR and CEA could increase the diagnostic efficacy for I-III stage CRC. J Clin Lab Anal. 2016 Sept 30. doi: 10.1002/jcla.22075. [Epub ahead of print] 